\title{
Review of: "C-Glycoside metabolism in the gut and in nature: Identification, characterization, structural analyses and distribution of C-C bond-cleaving enzymes"
}

\author{
Agnieszka Bartmańska
}

Potential competing interests: I declare no competing interests

In this very extensive study, the authors characterized two deglycolising enzymes (CGDs) derived from gut bacteria and three from soil bacteria. Enzymes have been isolated through assimilation screening and genome exploration.

Detailed biochemical analysis of all tested enzymes showed that they catalyzed the selective cleavage of C-C bonds in 3'-oxo-C6-glycosylated or C8-glycosylated substrates, while showing broad substrate specificity toward the aglycon structures.

Moreover, SEC-MALS analysis showed that the CGDs of intestinal bacteria were proteins with an $\alpha 4 \beta 4$ heterooctamer structure, while the enzymes of soil bacteria had an $\alpha \beta$ heterodimer structure.

Based on detailed X-ray analysis of crystal structures supported by Protein Data Bank data, further biochemical analyzes and targeted mutagenesis, the C-C bond cleavage mechanism was proposed as a general metal-assisted acid-base mechanism common to the CDG enzymes tested.

My minor comments:

1. All Latin names should be italicized, e.g. Actinobacteria, Proteobacteria, in vitro

2. Instead of phyla I would use genus

3. Minor bibliographic errors such as periods, spaces, e.g. Microbiol, ChemBioChem

4. In my opinion, the optimistic conclusions of the authors: „Because the C-C bond cleavage is crucial for Cglycosides to exert their biological activities, these findings will facilitate clarification of the bio-availability of xenobiotic C-glycosides in humans and the biogeochemical circulation of C-glycosides in nature" are an incentive today to extend the research to other CGD enzymes. 